ORIGINAL ARTICLE

\title{
Dorsalis pedis arterial pulse: palpation using a bony landmark
}

\author{
A Mowlavi, J Whiteman, B J Wilhelmi, M W Neumeister, R McLafferty
}

Postgrad Med J 2002;78:746-747

\begin{abstract}
Introduction: The unreliability of the pulse examination of the foot has primarily been due to variability of technique between examiners. Whereas the groove between the medial malleolus and the Achilles tendon more readily defines the location of the posterior tibial pulse, the location of the dorsalis pedis pulse remains vague. In this paper a novel method of locating the dorsalis pedis pulse by physical examination is described.

Methods: Forty one consecutive patients admitted to a general surgery service of a tertiary medical centre within a two month period were examined. Using the dorsal most prominence of the navicular bone as a landmark, the distance to the dorsalis pedis pulse in bilateral lower extremities was measured by palpation and compared to Doppler ultrasound. Measurements were confirmed by two separate examiners blinded to each others' results.

Results: The dorsalis pedis artery was palpable in $78 \%$ of extremities and present by Doppler ultrasound in $95 \%$. The location of the left dorsalis pedis artery was a mean (SD) 9.8 (1.4) mm by palpation and $11.1(2.1) \mathrm{mm}$ by Doppler ultrasound from the dorsal most prominence of the navicular bone. The right dorsalis pedis artery was $10.4(3.4) \mathrm{mm}$ by palpation and $11.5(0.7) \mathrm{mm}$ from the dorsal most prominence of the navicular bone. No significant differences in location of the dorsalis pedis artery were observed bilaterally between Doppler ultrasound and palpation; No significant differences were observed comparing contralateral dorsalis pedis arteries nor any differences between the examiners' results.

Conclusion: The dorsal most prominence of the navicular bone provides a bony landmark to readily locate the dorsalis pedis artery. Reliability of the examination may be increased as to the patency of
\end{abstract}

See end of article for authors' affiliations

Correspondence to:

Assistant Professor Bradon

Assistant Professor Bracthelmi, 747 N

Rutledge, P O Box 19230,

Springfield, Illinois

62794-1511, USA:

bwilhelmi@siumed.edu

Submitted 6 March 2002 Accepted 31 July 2002 the dorsalis pedis artery by using this dependable anatomic landmark.
$\mathrm{E}$ xamination of pedal pulses remains a useful clinical tool when evaluating peripheral circulation. Although a correlation between the ability to palpate pulses and the degree of systolic perfusion pressure has been established, controversy surrounds its accuracy. ${ }^{1}$ Unreliance among investigators stems from discrepancies related to arterial size, amount of subcutaneous fat, oedema, or neurovascular diabetic changes. ${ }^{23}$ Investigator variability is also a confounder of accurate pulse detection and includes factors such as fingertip sensitivity, anatomic knowledge, or sensation of the examiner's own fingertip pulsation. ${ }^{4}$ In fact, accuracy of pulse palpation has been most associated with level of the examiner's experience. ${ }^{5}$

We have observed increased variability among house staff when examining for the dorsalis pedis arterial pulse when compared with the posterior tibial arterial pulse. A specific landmark, notably a groove between the medial malleolus and the Achilles tendon, is described for the palpation of the posterior tibial pulse. ${ }^{6}$ In contrast, only a soft tissue boundary as defined medially by the extensor hallucis longus tendon running over the dorsum of the foot, has been defined as a guide for the location of the dorsalis pedis artery. Since the extensor hallucis longus tendon maintains a lengthy course, this landmark provides a vague guideline for palpation of the dorsalis pedis pulse. Instead, we chose the dorsal most prominence of the navicular bone as our bony landmark since this is the most prominent aspect of the dorsum of the foot. The nearest distance between the dorsal most prominence of the navicular bone and dorsalis pedis pulse was measured while palpating in a posterior-lateral direction towards the lateral malleolus in an attempt to define a more reliable bony anatomical landmark.

\section{SUBJECTS AND METHODS}

Forty one random, consecutive subjects who were admitted to the hospital under the care of the surgical team were selected as our subjects. The location of the dorsalis pedis artery pulse was recorded using two finger palpation and subsequently verified using a handheld Doppler. The dorsal most prominence of the navicular bone was marked (fig 1). Pulse palpation was evaluated by using two fingers, the index and middle fingers of the dominant hand (fig 1). Attempted detection of the pulse was initiated at the dorsal most prominence of the navicular bone and carried out following an arc over the dorsum of the foot towards the lateral malleolus in a posteriorlateral direction (fig 2). Palpable pulses and Doppler signal distances were measured from the tuberosity of the navicular bone using a surgical caliper. Pulse palpation and Doppler signal locations were measured blindly by two independent examiners (a fourth year medical student and third year plastic surgery resident). These distances were tabulated and statistical analysis using Student's $t$ test was completed by a staff statistician using Jandel Scientific (version 2.0, San Rafael, CA).

\section{RESULTS}

We present descriptive analysis of the location of the dorsalis pedis arterial pulse as measured from the dorsal most prominence of the navicular bone in 41 hospital subjects. The age of the patients ranged from 21 to 94 years old with a mean (SD) of 65 (20) years. Patients included 28 females and 13 males and had a wide range of past medical histories. Of the 82 pulses evaluated, detection was completed in 64 (78\%) by palpation and in $78(95 \%)$ by Doppler signal. No difference was detected in the location of the left dorsalis pedis arterial pulse 


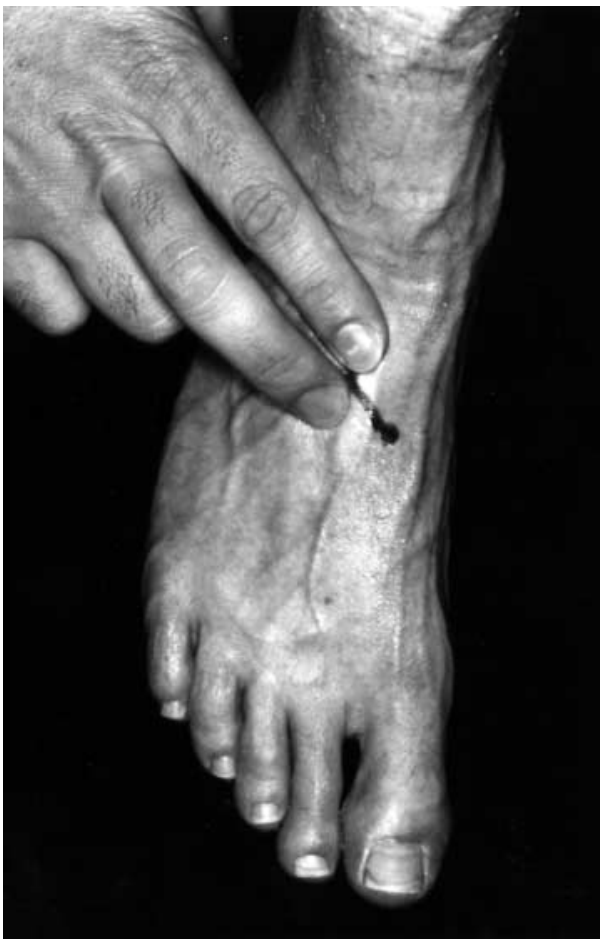

Figure 1 The tuberosity of the navicular bone was marked. Pulse palpation was performed using two fingers, the index and middle fingers of the dominant hand.

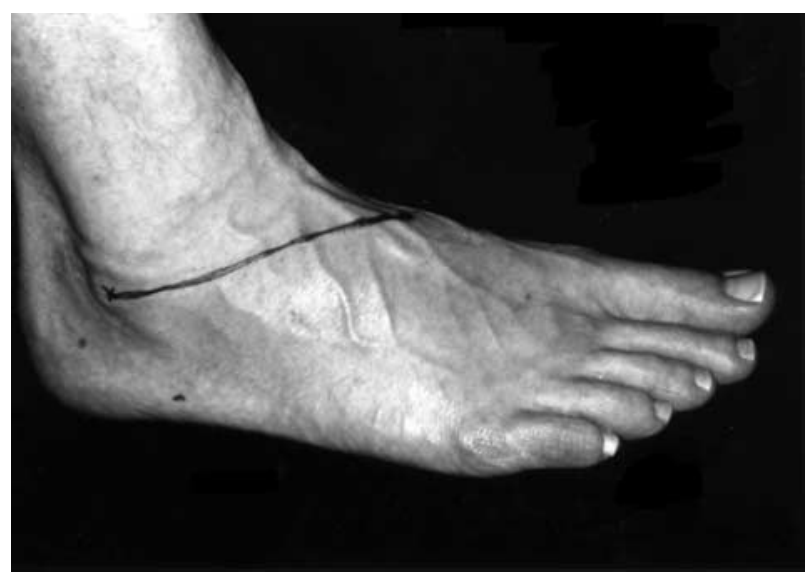

Figure 2 Attempted detection of the pulse was initiated at the tuberosity of the navicular bone and carried out following an arc over the dorsum of the foot towards the lateral malleolus in a posterior-lateral direction.

when located by palpation at a mean (SD) of 9.8 (1.4) $\mathrm{mm}$ versus 11.1 (2.1) $\mathrm{mm}$ by Doppler signal $(\mathrm{p}=0.247)$. No difference was detected in the location of the right dorsalis pedis arterial pulse when located by palpation at $10.4(3.4)$ mm versus $11.5(0.7) \mathrm{mm}$ by Doppler signal $(\mathrm{p}=0.305)$. No difference was detected between contralateral pulse locations when detected by palpation ( $\mathrm{p}=0.528$ ) nor when detected by Doppler signal $(p=0.731)$ and no difference was observed between independent examiners' results $(p>0.05)$.

\section{DISCUSSION}

We have demonstrated a quick and reliable method for locating the dorsalis pedis arterial pulse by palpation and/or Doppler ultrasound. The dorsalis pedis artery is a continuation of the anterior tibial artery distal to the ankle anteriorly. Distal to the ankle, the dorsalis pedis artery travels lateral to the extensor hallucis tendon along its course to the great toe. ${ }^{7}$ Previous studies have reported the dorsalis pedis pulse impalpable in $3.1 \%$ to $13.8 \%$ of healthy patients. ${ }^{58}$ Sensitivity of palpation has been proposed to be increased by avoidance of plantar flexion. ${ }^{7}$ Plantar flexion of the foot is believed to increase tension of the skin along the extensor hallucis tendon thus obliterating an underlying pulse. ${ }^{7}$ Alternatively, it is possible that tensing the skin with plantar flexion results in raising of the vessel within the subcutaneous tissue from its immediate contact with the bony dorsum making palpation more difficult. Doppler examination of pedal pulses is generally accepted as more accurate than pulse palpation. ${ }^{5}$ As such, Doppler ultrasound examination typically demonstrates increased sensitivity with an absent signal over the presumed location of the dorsalis pedis artery in only $1.9 \%$ of patients.

Discrepancy of pedal pulse detection and with regards to its accuracy persists among clinicians. Since the location of the posterior tibial artery is well delineated by a bony landmark, it is more reliably detected by Doppler ultrasonography. One study demonstrated increased interinvestigator agreement among $78 \%$ of posterior tibial pulses versus only $58 \%$ of the dorsalis pedis pulses when identified by Doppler ultrasonography. ${ }^{9}$ Paradoxically, the posterior tibial artery is believed to be more difficult to palpate in comparison with the dorsalis pedis artery. ${ }^{10}$ This is felt to be secondary to the deeper location of the posterior tibial artery especially when compounded by ankle swelling. ${ }^{9}$ In contrast, the dorsalis pedis arterial pulse is felt to be more accessible to finger palpation since it lies in the immediate subcutaneous tissue on the dorsum of the foot. ${ }^{8}$

Pedal pulse detection remains a mainstay for lower extremity perfusion assessment; thus, establishing exact pulse detection techniques are necessary. Although the dorsalis pedis arterial pulse detection is relied upon for perfusion assessment, its location is vaguely described and not associated with a definitive bony landmark. We present a quick and reliable method for assessment of the dorsalis pedis pulse utilising the dorsal most prominence of the navicular bone. The dorsalis pedis pulse can be detected to within 1 to $1.5 \mathrm{~cm}$ of this bony landmark using palpation or Doppler ultrasonography. We feel that utilising our presented technique will facilitate a more efficient means of detecting pedal pulses.

\section{Authors' affiliations}

A Mowlavi, J Whiteman, B J Wilhelmi, M W Neumeister, $R$ McLafferty, Southern Illinois University, The Plastic Surgery Institute, Springfield, Illinois, USA

\section{REFERENCES} 1 Brearley S, Simms MH, Shearman CP. Peripheral pulse palpation: an

2 Goss DE, de Trafford JC, Roberts VC, et al. Raised ankle/brachial index in insulin treated diabetic patients. Diabet Med 1989;6:576-8.

3 Quigley FG, Faris IB, Duncan HJ. A comparison of Doppler ankle pressures and skin perfusion pressure in subjects with and without diabetes. Clin Physiol 1991;1:21-5.

4 Lundin M. Wiksten J-P, Perakyla T, et al. Distal pulse palpation: is it reliable? World J Surg 1999;23:252-5.

5 Meade TW, Gardner M, Cannon P, et al. Observer variability in recording the peripheral pulses. Br Heart J 1968;30:661-5.

6 DeGowin RL. DeGowin \& Degowin's diagnostic examination. 6th Ed. St Louis, MO: McGraw-Hill, 1994: 411-16.

7 Ison JW. Palpation of dorsalis pedis pulse. JAMA 1968;206:2745

8 Ludbrook J. Clarke AM, McKenzie JK. Significance of absent ankle pulse. BM 1962;i: 1724-6.

9 Magee TR, Stanley PRW, Mufti RA. Should we palpate foot pulses? Ann $R$ Coll Surg Engl 1992;74:166-8.

10 Lawson IR, Ingman SR, Masih Y, et al. Reliability of palpation of pedal pulses as ascertained by the kappa statistic. J Am Geriatr Soc 1980:28:300-3. 